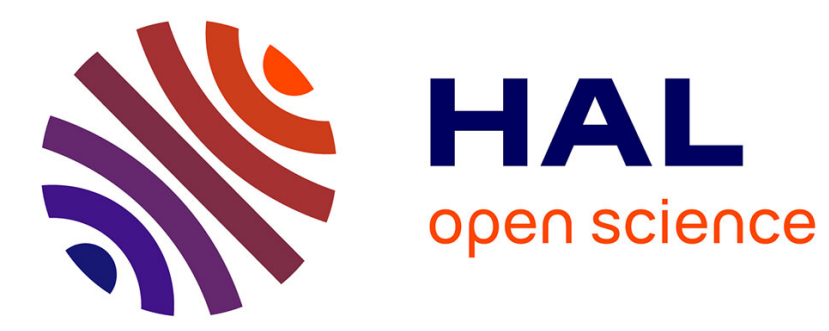

\title{
Lagrangian data assimilation for river hydraulics simulations
}

Marc Honnorat, Jerome Monnier, François-Xavier Le Dimet

\section{To cite this version:}

Marc Honnorat, Jerome Monnier, François-Xavier Le Dimet. Lagrangian data assimilation for river hydraulics simulations. Computing and Visualization in Science, 2009, 12 (5), pp.235-246. 10.1007/s00791-008-0089-x . inria-00256581

\section{HAL Id: inria-00256581 https://hal.inria.fr/inria-00256581}

Submitted on 15 Feb 2008

HAL is a multi-disciplinary open access archive for the deposit and dissemination of scientific research documents, whether they are published or not. The documents may come from teaching and research institutions in France or abroad, or from public or private research centers.
L'archive ouverte pluridisciplinaire HAL, est destinée au dépôt et à la diffusion de documents scientifiques de niveau recherche, publiés ou non, émanant des établissements d'enseignement et de recherche français ou étrangers, des laboratoires publics ou privés. 


\section{Marc Honnorat • Jérôme Monnier • François-Xavier Le Dimet \\ Lagrangian data assimilation for river hydraulics simulations}

Received: date / Accepted: date

\begin{abstract}
We present a method to use lagrangian data from remote sensing observation in a variational data assimilation process for a river hydraulics model based on the bidimensional shallow water equations. The trajectories of particles advected by the flow can be extracted from video images and are used in addition to classical eulerian observations. This lagrangian data brings information on the surface velocity thanks to an appropriate transport model. Numerical twin data assimilation experiments in an academic flow configuration demonstrate that this method makes it possible to significantly improve the identification of local bed elevation and initial conditions.
\end{abstract}

\section{Introduction}

The numerical simulation of river flows requires a precise modelling of the underlying physics. The bidimensional shallow water equations can describe accurately many free surface hydraulic configurations. In order to carry out a realistic simulation of a particular system, numerical models require information on physical parameters such as bed elevation, roughness coefficients in addition to initial and boundary conditions. Unfortunately, many model parameters are usually not well known and must be calibrated. Since the quality of the simulation is largely dependent on these model inputs, the latter must be defined accurately.

To improve the quality of the simulation, data assimilation methods combine optimally information from the model and observation data in order to identify the value of model parameters consistent with reality. Variational data assimilation [15] consists in minimizing a cost function that measures the discrepancy between simulation results and physical measurements. The minimization can be performed by a quasi-Newton method (see

M. Honnorat · J. Monnier · F.-X. Le Dimet LMC-IMAG, B.P. 53, 38041 Grenoble Cedex 9, France

E-mail: marc.honnorat@imag.fr e.g. [7]), which requires the computation of the gradient of the cost function. The latter can be efficiently computed using the solution to an adjoint model.

Data assimilation methods have been used in river model for the identification of model parameters in one dimensional channels [13,22], using Kalman filter $[9,18]$ or for bidimensional shallow water equations using variational methods $[1,2,6,19,26]$.

However, in river hydraulics, observation data is available only in very small quantities. River water level can be measured locally at gauging stations, but such observations are usually very sparse in space. Velocity measurements are even more scarce, since they usually require complex human interventions. Consequently, the available eulerian observations can be insufficient to take full advantage of data assimilation for many identification problems.

Therefore, new kinds of observations would bring additional information on the flow. In particular, remote sensing techniques make it possible to get information on the water surface $[5,20]$. In oceanography, data assimilation of drifting buoys positions has been experimentally used to improve numerical ocean models, either using Kalman filter $[3,14,23]$ or variational based methods (see [12] for a shallow water model and more recently [21] for a primitive equations circulation model of the ocean).

In this article, we present a method to use lagrangian observations of particles spread on the surface of water and advected by the river flow, into a variational data assimilation procedure. The link between trajectories of the particles, which can be extracted from video images, and the shallow water model is made thanks to an appropriate transport model. We introduce a cost function measuring the distance between the trajectory of model particles and the available observations. Lagrangian data is used in addition to classical eulerian observations of water depth.

When considering real flows, the water surface is perturbed by physical phenomena not included in the physics of the model, such as small-scale turbulence, secondary currents, etc. To cope with this difficulty, an observa- 
tion operator based on a simple spatio-temporal filtering scheme is proposed to remove small-scale perturbations from trajectories observations.

Lagrangian data assimilation is applied to the identification of bed elevation and initial conditions for an academic configuration of a river hydraulics model based on the bidimensional shallow water equations. The method is implemented in a software called Dassflow [11] designed to carry out data assimilation experiments. The discretization of the direct shallow water model relies on the finite volume method and the HLLC [25] approximate Riemann solver. The adjoint model is written using the automatic differentiation tool Tapenade [10].

Numerical twin data assimilation experiments are carried out using synthetic observations of trajectories generated by a turbulent velocity field where small scale perturbations are modelled by a Gauss-Markov stochastic process. These experiments demonstrate that the proposed method makes it possible to improve significantly the quality of the identification.

We introduce the shallow water equations in Section 2 and the variational data assimilation procedure for lagrangian data in Section 3. The discretization of the direct model, the numerical scheme for the computation of trajectories and the implementation of the adjoint model are presented in Section 4. A filter used to smooth observations of trajectories is described in Section 5. Finally, twin experiments and numerical results of data assimilation for the identification of bed elevation and initial conditions are presented in Sections 6 and 7.

\section{Shallow Water model}

The river hydraulics model considered relies on the bidimensional shallow water equations in a conservative formulation. The state variables are the water depth $h$ and the local discharge $\mathbf{q}=h \mathbf{u}$, where $\mathbf{u}$ is the depth-averaged velocity vector. On a domain $\Omega$ and for a computational time interval $[0, T]$, the shallow water equations associated with initial and boundary conditions can be written as

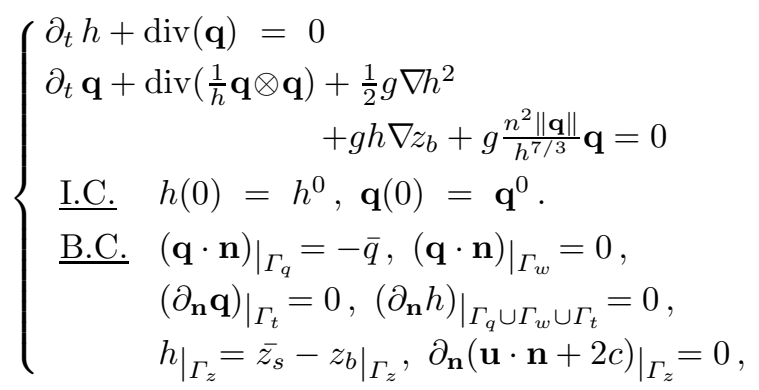

where $g$ is the magnitude of the gravity, $z_{b}$ the bed elevation, $n$ the Manning roughness coefficient, $h^{0}$ and $\mathbf{q}^{0}$ the initial conditions for the state variables. The variable $c=\sqrt{g h}$ denotes the local wave celerity.
The boundary $\Gamma$ of the domain $\Omega$ is split up into four different kinds of boundary conditions $\Gamma=\Gamma_{q} \cup \Gamma_{z} \cup \Gamma_{t} \cup$ $\Gamma_{w}$. Boundaries $\Gamma_{q}, \Gamma_{z}$ and $\Gamma_{t}$ correspond to open (fluidto-fluid) boundaries while $\Gamma_{w}$ corresponds to a wall.

$-\Gamma_{q}$ : a scalar discharge $\bar{q}$ is prescribed

$-\Gamma_{z}$ : a water elevation $\overline{z_{s}}$ is prescribed and the Riemann invariant is constant along the outgoing characteristic

- $\Gamma_{t}$ : homogeneous Neumann conditions for all state variables are prescribed

$-\Gamma_{w}$ : a slip condition on the velocity is prescribed.

Boundary condition on $\Gamma_{z}$ is valid only for sub-critical flows, i.e. when $\|\mathbf{u}\|<c$. Otherwise, homogenous Neumann conditions are prescribed.

The model state variables $(h, \mathbf{q})$ are completely determined by the value of model parameters, initial conditions and boundary conditions gathered in the control vector $\mathbf{k}=\left(h^{0}, \mathbf{q}^{0}, n, z_{b}, \bar{q}, \overline{z_{s}}\right)$.

\section{Lagrangian Data Assimilation}

Variational data assimilation $[15,24]$ is based on optimal control theory [17] and consists in identifying the control vector $\mathbf{k}$ that minimizes a cost function measuring the discrepancy between the state variable of the model and data obtained from the observation of the physical system. An efficient minimization of the cost function is carried out using a quasi-Newton method that requires the computation of its gradient. We use the M1QN3 algorithm [7] based on the BFGS formula.

Lagrangian data assimilation consists in using observations described by lagrangian coordinates in the data assimilation process. Here, we consider observations of passive particles transported by the river flow. However, the state of the flow is described in eulerian coordinates by the shallow water model. The link between the lagrangian data and the classical eulerian variables of the shallow water model is made by an appropriate transport model.

\subsection{Transport model}

Let us consider a set of $N$ particles transported by the flow. We state that their trajectories $X_{i}(t)$ are solutions of the following ODEs:

$$
\begin{aligned}
& \text { For } i=1, \ldots, N: \\
& \left\{\begin{array}{l}
\frac{d}{d t} X_{i}(t)=\mathbf{v}\left(X_{i}(t), t\right) \\
X_{i}\left(t_{i}^{0}\right)=x_{i}^{0}
\end{array} \quad \forall t \in\right] t_{i}^{0}, t_{i}^{f}[
\end{aligned}
$$

where $\mathbf{v}$ is the transport velocity of the particles, $t_{i}^{0}$ and $t_{i}^{f}$ are the time when the particle enters and leaves the 
observation domain. In the following, the transport velocity is related to the shallow water model velocity by a multiplicative constant: $\mathbf{v}=\gamma \mathbf{u}$.

This set of ODEs is weakly coupled with the shallow water model since the state variable of the latter is not dependent on the solution of the former.

\subsection{Observations and cost function}

We consider two kinds of observations. The first one consists in classical, eulerian observations of the water depth in some locations of the physical domain, denoted by $h^{\text {obs }}(t)$. The second kind consists in the trajectories of physical particles transported by the water flow. These lagrangian observations are denoted by $X_{i}^{\text {obs }}(t)$.

In order to take into account both kinds of observations, we build a composite cost function measuring the discrepancy between observation data and model state variables:

$$
\begin{aligned}
j(\mathbf{k}) & =\frac{1}{2} \int_{0}^{T}\left\|C h(t)-h^{o b s}(t)\right\|_{o b s}^{2} d t \\
& +\frac{\alpha_{t}}{2} \sum_{i=1}^{N} \int_{t_{i}^{0}}^{t_{i}^{f}}\left|X_{i}(t)-X_{i}^{o b s}(t)\right|^{2} d t \\
& +\frac{\alpha_{p}}{2}\left\|D z_{b}\right\|_{k}^{2}
\end{aligned}
$$

where $\mathbf{k}=\left(h^{0}, \mathbf{q}^{0}, n, z_{b}, \bar{q}, \overline{z_{s}}\right)$ is the control vector, $\alpha_{t}$ and $\alpha_{p}$ scaling parameters. The operator $C$ computes the restriction of the model state variable $h$ to the space of eulerian observations on which is defined the norm $\|\cdot\|_{o b s} . D$ is a differential operator and $\|\cdot\|_{k}$ is a norm defined on the space of controls. The first term measures the discrepancy between water depth observations and model state variable. The second term measures the distance between virtual particles of the transport model and observations of trajectories. The third one is a regularization term on the bed elevation.

\subsection{Adjoint model}

The adjoint method [17] makes it possible to compute efficiently all partial derivatives of the cost function $j$ with respect to the components of the control vector $\mathbf{k}$. We introduce the following weakly coupled adjoint model set.

$$
\left\{\begin{array}{cl}
\frac{\text { For } i=1, \ldots, N}{d \widetilde{X}_{i}(t)+\gamma[\nabla \mathbf{u}]^{T} \widetilde{X}_{i}(t)=} & \\
\alpha_{t}\left(X_{i}(t)-X_{i}^{o b s}(t)\right) & \forall t \in] t_{i}^{0}, t_{i}^{f}[ \\
\tilde{X}_{i}(t)=0 & \left.\forall t \in] 0, t_{i}^{0}\right] \cup\left[t_{i}^{f}, T[\right.
\end{array}\right.
$$

$$
\left\{\begin{aligned}
& \partial_{t} \widetilde{h}-[(\mathbf{u} \cdot \nabla) \widetilde{\mathbf{q}}] \cdot \mathbf{u}+g h \operatorname{div}(\widetilde{\mathbf{q}}) \\
&-g \widetilde{\mathbf{q}} \cdot \nabla z_{b}+\frac{7}{3} g \frac{n^{2}\|\mathbf{u}\|}{h^{4 / 3}} \mathbf{u} \cdot \widetilde{\mathbf{q}} \\
&=\frac{\gamma}{h} \sum_{i=1}^{N} \mathbf{u} \cdot \widetilde{X}_{i}+C^{T}\left(C h-h^{o b s}\right) \\
& \partial_{t} \widetilde{\mathbf{q}}+\nabla \widetilde{h}+(\mathbf{u} \cdot \nabla) \widetilde{\mathbf{q}}+(\nabla \widetilde{\mathbf{q}})^{T} \mathbf{u} \\
&-g \frac{n^{2}\|\mathbf{u}\|}{h^{4 / 3}} \widetilde{\mathbf{q}}-g \frac{n^{2}}{h^{4 / 3}\|\mathbf{u}\|}(\mathbf{u} \otimes \mathbf{u}) \widetilde{\mathbf{q}} \\
&=-\frac{\gamma}{h} \sum_{i=1}^{N} \widetilde{X}_{i}
\end{aligned}\right.
$$$$
\begin{cases}\underline{\text { I.C. }} & \widetilde{h}(T)=0, \quad \widetilde{\mathbf{q}}(T)=0, \\ \underline{\text { B.C. }} & \left.\widetilde{\mathbf{q}}\right|_{\Gamma_{q}}=0,\left.(\widetilde{\mathbf{q}} \cdot \mathbf{n})\right|_{\Gamma_{w}}=0,\left.\widetilde{\mathbf{q}}\right|_{\Gamma_{t}}=0, \\ & \left.(\widetilde{\mathbf{q}} \cdot \tau)\right|_{\Gamma_{z}}=0,\left.(\widetilde{h}+2(\mathbf{u} \cdot \mathbf{n})(\widetilde{\mathbf{q}} \cdot \mathbf{n}))\right|_{\Gamma_{z}}=0 \\ & \left.\left(\partial_{\mathbf{n}} \widetilde{h}\right)\right|_{\Gamma_{q} \cup \Gamma_{w}}=0,\left.\widetilde{h}\right|_{\Gamma_{t}}=0 .\end{cases}
$$

A backward integration in time of the adjoint transport model (4) followed by a backward integration in time of the adjoint shallow water model (5)-(6) give a solution $(\tilde{X}, \widetilde{h}, \widetilde{\mathbf{q}})$ to the weakly coupled system. Then, the partial derivatives of the cost function are simple functions of the adjoint state variables $\widetilde{h}$ and $\widetilde{\mathbf{q}}$. We have

$$
\begin{aligned}
& \frac{\partial j}{\partial h^{0}}(\mathbf{k})=-\widetilde{h}(0), \quad \frac{\partial j}{\partial \mathbf{q}^{0}}(\mathbf{k})=-\widetilde{\mathbf{q}}(0), \\
& \frac{\partial j}{\partial z_{b}}(\mathbf{k})=\alpha_{p} D^{T} D z_{b}-\int_{0}^{T} \operatorname{div}(g h(t) \widetilde{\mathbf{q}}(t)) d t, \\
& \frac{\partial j}{\partial n}(\mathbf{k})=2 g n \int_{0}^{T}\|\mathbf{u}(t)\| h(t)^{-\frac{1}{3}} \mathbf{u}(t) \cdot \widetilde{\mathbf{q}}(t) d t, \\
& \frac{\partial j}{\partial \bar{q}}(\mathbf{k})=-\left.\widetilde{h}\right|_{\Gamma_{q}}, \\
& \frac{\partial j}{\partial \bar{z}_{s}}(\mathbf{k})=\left.\left[(\widetilde{\mathbf{q}} \cdot \mathbf{n})\left(c^{2}-(\mathbf{u} \cdot \mathbf{n})^{2}\right)\right]\right|_{\Gamma_{z}} .
\end{aligned}
$$

These partial derivatives are used as inputs to the minimization algorithm. A single integration of the direct model (1)-(2) followed by a single integration backward in time of the adjoint model (4)-(6) are sufficient to compute all components of the gradient of the cost function.

\section{Discretization}

\subsection{Finite Volume solver}

The bidimensional shallow water equations implemented in Dassflow [11] are solved numerically on an unstructured mesh using the finite volume method. The system (1) can be written in a general form as

$$
\partial_{t} U+\operatorname{div} F(U)=S(U)
$$

where $U=(h, \mathbf{q})^{T}$ is the vector of conservative variables, $F(U)=(G(U), H(U))^{T}$ the flux vector and $S(U)$ the 
source term. The expression of these vectors, noting $\mathbf{q}=$ $\left(q_{x}, q_{y}\right)^{T}$, is the following

$$
\begin{gathered}
G(U)=\left(\begin{array}{c}
q_{x} \\
\frac{1}{h} q_{x}^{2}+\frac{1}{2} g h^{2} \\
\frac{1}{h} q_{x} q_{y}
\end{array}\right), \quad H(U)=\left(\begin{array}{c}
q_{y} \\
\frac{1}{h} q_{x} q_{y} \\
\frac{1}{h} q_{y}^{2}+\frac{1}{2} g h^{2}
\end{array}\right), \\
S(U)=\left(\begin{array}{c}
0 \\
-g h \partial_{x} z_{b}-g \frac{n^{2}\|\mathbf{q}\|_{2}}{h^{7 / 3}} q_{x} \\
-g h \partial_{y} z_{b}-g \frac{n^{2}\|\mathbf{q}\|_{2}}{h^{7 / 3}} q_{y}
\end{array}\right) .
\end{gathered}
$$

The computational domain $\Omega$ is discretized using triangular and quadrangular cells. We define the mean value of the state variable $U$ on an arbitrary cell $K_{i}$ (see Fig. 1) by

$$
U_{i}=\frac{1}{\left|K_{i}\right|} \int_{K_{i}} U d \Omega
$$

where $\left|K_{i}\right|$ denotes the surface of the cell. By integrating (7) over $K_{i}$, using the divergence theorem, we obtain

$$
\int_{K_{i}} \partial_{t} U d \Omega+\sum_{j=1}^{N_{i}} \int_{E_{i j}} T_{i j}^{-1} G\left(T_{i j} U\right) d s=\int_{K_{i}} S(U) d \Omega
$$

where $N_{i}$ denotes the number of faces of the cell $K_{i}$ (3 or 4), $E_{i j}$ is the cell interface and $T_{i j}$ is the $3 \times 3$ rotation matrix of angle $\theta_{i j}$ between the horizontal plane and the normal to $E_{i j}$. The usual flux term derived from the di-

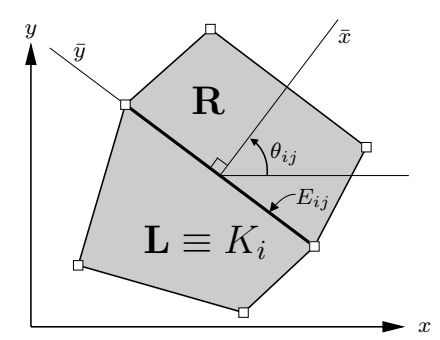

Fig. 1 Two adjacent finite volumes.

vergence theorem has been replaced by integrals over the cell edges thanks to the rotational invariance property of the shallow water equations (see [25, p. 65]). Hence, the bidimensional problem actually consists in a sum of onedimensional local Riemann problems that can be solved numerically using a Riemann solver. One can write the following semi-discrete scheme

$$
\frac{d}{d t} U_{i}+\frac{1}{\left|K_{i}\right|} \sum_{j=1}^{N_{i}} T_{i j}^{-1} \widetilde{G}\left(U_{L}, U_{R}\right)=S_{i}
$$

where $\widetilde{G}\left(U_{L}, U_{R}\right)$ is an approximation of the flux through the cell interface $E_{i j}$. Subscripts $L$ and $R$ denote cells respectively to the left and to the right of the interface.
To compute numerically the discrete flux $\widetilde{G}\left(U_{L}, U_{R}\right)$, we use the HLLC approximate Riemann solver [25]. The discretization of the bed slope is actually included in the flux term so that the balance between the source term and the fluxes is preserved for some steady state solutions [16]. A forward Euler scheme is used for time discretization. In order to ensure stability, the following stability condition on the time step $\Delta t$ must be satisfied

$$
\Delta t \leq \frac{\min \left(d_{L, R}\right)}{\max (\|\mathbf{u}\|+c)}
$$

where $d_{L, R}$ is the distance between the cell center and the center of interface (see [25, p. 157]).

In the following, we shall consider only structured regular meshes with quadrangles, the horizontal and vertical space discretization intervals being denoted respectively by $h_{x}$ and $h_{y}$. Hence we have $d_{L, R}=\frac{1}{2} \min \left(h_{x}, h_{y}\right)$.

\subsection{Trajectories}

The numerical trajectories of the particles are obtained by the integration of the transport equation (2) using a second-order Runge-Kutta scheme. Let $\left(t^{n}\right)_{n}$ be a subdivision of the time interval $\left[t_{i}^{0}, t_{i}^{f}\left[\right.\right.$ and let $\Delta t^{n}=t^{n+1}-t^{n}$. An approximation $X_{i}^{n+1}$ of the position $X_{i}\left(t^{n+1}\right)$ of particle number $i$ at time $t^{n+1}$ is given by

$$
\begin{aligned}
& X_{i}^{0}=x_{i}^{0} \\
& \text { For } n=0,1, \ldots: \\
& \left\{\begin{array}{c}
X_{i}^{n, 1}=X_{i}^{n}+\Delta t^{n} \mathbf{v}\left(X_{i}^{n}, t^{n}\right) \\
X_{i}^{n, 2}=X_{i}^{n}+\Delta t^{n} \mathbf{v}\left(X_{i}^{n, 1}, t^{n+1}\right) \\
X_{i}^{n+1}=\frac{1}{2}\left(X_{i}^{n, 1}+X_{i}^{n, 2}\right)
\end{array}\right.
\end{aligned}
$$

This scheme requires the computation of the velocity for an arbitrary position $P=\left(p_{x}, p_{y}\right)$ in the domain $\Omega$. However, the velocity $\mathbf{v}$ is known only as a constant piecewise field, solution to the finite volume discretization of the shallow water equations. Let $\mathbf{v}_{j, k}^{n}$ denote the approximation of its mean value on each cell $K_{j, k}$ at time $t^{n}$. Using notations of Fig. 2, we calculate a Q1-Lagrange interpolation $\mathbf{v}_{P}\left(t^{n}\right)$

$$
\begin{aligned}
\mathbf{v}_{P}\left(t^{n}\right)=\frac{1}{h_{x} h_{y}} & \left(\left(h_{x}-\Delta x\right)\left(h_{y}-\Delta y\right) \mathbf{v}_{j, k}^{n}\right. \\
& +\Delta x \Delta y \mathbf{v}_{j+1, k+1}^{n} \\
& +\Delta x\left(h_{y}-\Delta y\right) \mathbf{v}_{j+1, k}^{n} \\
& \left.+\left(h_{x}-\Delta x\right) \Delta y \mathbf{v}_{j, k+1}^{n}\right)
\end{aligned}
$$

Since the mesh is structured and the cells are rectangular, this scheme is second-order accurate. 


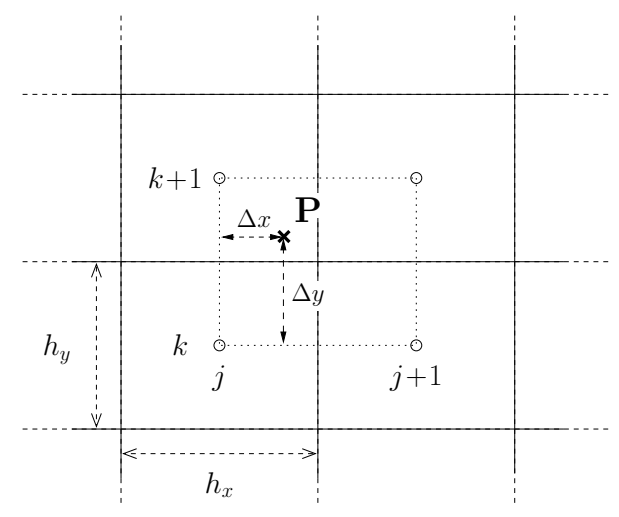

Fig. 2 Interpolation grid on a structured, rectangular mesh of the computational domain for the velocity field.

\subsection{Adjoint solver}

In practice, there are three main methods to obtain an implementation of the adjoint model. The continuous adjoint model (4)-(6) can be discretized using an appropriate numerical scheme which is then implemented. A second solution consists in writing the adjoint of the direct numerical scheme and implementing it. Actually, we use a better way that consists in writing directly the adjoint code of the implementation of the direct model. This ensures the coherence between the discretizations of the direct and adjoint models. A large part of this extensive task can be automated using algorithmic differentiation [8]. Here, the direct program is coded in Fortran 90 and we use the automatic differentiation tool Tapenade [10].

\section{Trajectory filtering}

When we consider real flows, surface velocity is perturbed by many physical phenomena that are not taken into account neither in the shallow water model nor in the particle transport model. Therefore, if a large number of trajectories observations is available, it should be profitable to filter this information in order to remove small-scale perturbations from the data set. Such a filter based on a priori information on the flow can improve the quality of data assimilation and consequently the quality of parameter identification.

In the proposed method, filtered trajectories reconstructed from a local average in time and space of the observed velocity field are used instead of the raw trajectories in the data assimilation process. More precisely, we seek to create a set $\left\{\bar{X}_{j}\right\}_{j=1, N_{m}}$ of trajectories defined as

$$
\left\{\begin{array}{l}
\left.\frac{d}{d t} \bar{X}_{j}(t)=\overline{\mathbf{u}}\left(\bar{X}_{j}(t), t\right) \quad \forall t \in\right] t_{j}^{0}, t_{j}^{f}[ \\
\bar{X}_{j}\left(t_{j}^{0}\right)=x_{j}^{0}
\end{array}\right.
$$

where $\overline{\mathbf{u}}$ is the local average velocity of the observed particles and $x_{j}^{0}$ the starting point of the filtered trajectory $j$. Let

$$
\overline{\mathbf{u}}_{j}(t)=\overline{\mathbf{u}}\left(\bar{X}_{j}(t), t\right)
$$

denote the velocity of filtered particle $j$ at time $t$. It is computed as the mean velocity observed at time $t$ on a time-space window $\mathcal{W}=\mathcal{W}_{t} \times \mathcal{W}_{\bar{X}_{j}(t)}$, where $\mathcal{W}_{t}$ denotes a temporal neighborhood of $t$ and $\mathcal{W}_{\bar{X}_{j}(t)}$ a spatial neighborhood of $\bar{X}_{j}(t)$. Using these notations, we define

$$
\overline{\mathbf{u}}_{j}(t)=\frac{1}{\mathbb{W}_{t}^{j}} \sum_{i=1}^{N_{o b s}} \int_{\mathcal{W}_{t}} \frac{d}{d s} X_{i}^{o b s}(s) \mathbb{1}_{X_{i}^{o b s}(s) \in \mathcal{W}_{\bar{X}_{j}(t)}} d s
$$

where

$$
\mathbb{W}_{t}^{j}=\sum_{i=1}^{N_{\text {obs }}} \int_{\mathcal{W}_{t}} \mathbb{1}_{X_{i}^{o b s}(s) \in \mathcal{W}_{\bar{X}_{j}(t)}} d s .
$$

In Fig. 3 is drawn a square spatial window for a particle located in $\bar{X}$ at time $t$. Two raw trajectories of observed particles are represented with dotted lines. The solid lines correspond to the part of the trajectories included in the time window $\mathcal{W}_{t}$ while the bold lines match the intersection of the latter with the space window, i.e. the parts of the trajectories actually taken into account in the computation of the local mean velocity $\overline{\mathbf{u}}_{j}(t)$. The filtered trajectories $\bar{X}_{j}$ are used in place of the raw observations $X_{i}^{o b s}$ in the cost function for data assimilation.

\section{Twin experiments}

\subsection{Description}

Twin experiments consist in data assimilation experiments where the observations are created by the model with a given set of parameters. This makes it possible to evaluate a data assimilation method with a complete knowledge of all parameters, with no dependence on external variables.

A reference simulation is performed using a known set of parameters $\mathbf{k}^{r e f}$. The resulting state variables, called $h^{r e f}$ and $\mathbf{u}^{\text {ref }}$ are used to create observations. Here, we

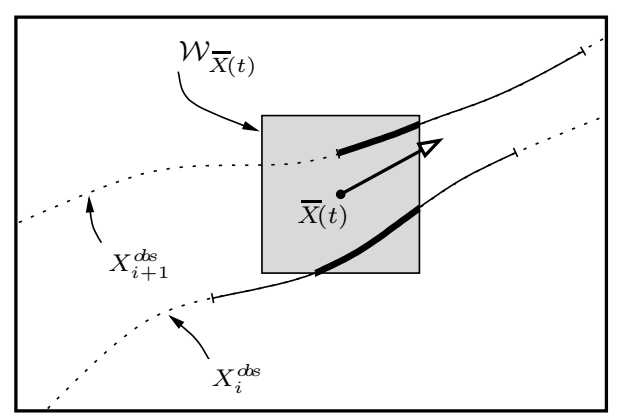

Fig. 3 Space and time windows for trajectories filtering. 
will consider observations of water depth, denoted by $h^{o b s}$ and observations of trajectories, denoted by $X^{o b s}$.

Then, a different configuration resulting from a modified set of parameters $\mathbf{k}^{0}$ made of a priori hypotheses on the reference flow is used as an initial guess for a data assimilation experiment. The aim is to identify the reference set of parameters $\mathbf{k}^{\text {ref }}$ using the synthetic observations.

In order to evaluate the quality of the identification, we introduce a diagnostic function $j_{\text {diag }}$ measuring the discrepancy between the reference state variable and a simulation resulting from an arbitrary parameter vector $\mathbf{k}$.

$$
\begin{aligned}
j_{\text {diag }}(\mathbf{k})=\frac{1}{2} \int_{0}^{T}( & \left\|h(t)-h^{r e f}(t)\right\|_{\Omega}^{2} \\
& \left.+\left\|\mathbf{u}(t)-\mathbf{u}^{r e f}(t)\right\|_{\Omega}^{2}\right) d t
\end{aligned}
$$

If the observations are perfect, directly derived from the model state variables with no additional noise, then we get $j_{\text {diag }}\left(\mathbf{k}^{r e f}\right)=0$.

\subsection{Perturbation of trajectories}

For numerical twin experiments, we will actually construct observations of perturbed trajectories, i.e. trajectories of particles transported by a turbulent velocity field denoted by $\mathbf{v}^{\text {tr }}$. We consider a simple perturbation model where the observations consist in trajectories of particles transported by a velocity field made up of the sum of the model velocity $\mathbf{v}=\gamma \mathbf{u}$ and a perturbation $\widetilde{\mathbf{v}}$.

$$
\mathbf{v}^{\operatorname{tr}}(x, t)=\mathbf{v}(x, t)+\widetilde{\mathbf{v}}(x, t) .
$$

We define the perturbation $\widetilde{\mathbf{v}}$ as a Gauss-Markov stochastic process. Let the random variable $\widetilde{\mathbf{v}}^{n}=\widetilde{\mathbf{v}}\left(\cdot, t^{n}\right)$ denote the perturbation velocity field at time $t^{n}$. We introduce the Reynolds tensor $R\left(t^{n}\right)$ and the time correlation tensor $\Lambda\left(t^{n}, t^{m}\right)$ defined as

$$
\begin{aligned}
R\left(t^{n}\right) & =\mathbb{E}\left(\widetilde{\mathbf{v}}^{n}\left(\widetilde{\mathbf{v}}^{n}\right)^{T}\right) \\
\Lambda\left(t^{n}, t^{m}\right) & =\mathbb{E}\left(\widetilde{\mathbf{v}}^{n}\left(\widetilde{\mathbf{v}}^{m}\right)^{T}\right) .
\end{aligned}
$$

The Reynolds tensor $R$ is a symmetric positive-definite matrix. We will consider the particular case where $\Lambda$ is an exponentially decreasing function in time of $R$

$$
\Lambda\left(t^{n}+\Delta t^{n}, t^{n}\right)=e^{-\frac{\Delta t^{n}}{T_{L}}} R\left(t^{n}\right),
$$

where $T_{L}$ is the local turbulence characteristic duration. The perturbation $\widetilde{\mathbf{v}}^{n}$ is defined by the following recursive equation

$$
\widetilde{\mathbf{v}}^{n+1}=e^{-\frac{\Delta t^{n}}{T_{L}}} \widetilde{\mathbf{v}}^{n}+\mathbf{w}^{n}
$$

where $\mathbf{w}^{n}$ is a random variable with a zero-mean gaussian distribution and a covariance matrix $W_{n}$ defined by

$$
W_{n}=\left(1-e^{-\frac{2 \Delta t^{n}}{T_{L}}}\right) R\left(t^{n}\right) .
$$

\section{Numerical results}

We present results of some twin experiments carried out to evaluate lagrangian data assimilation for a river hydraulics configuration. Observations of particle trajectories are used in combination with local water depth measurements, first for the identification of local bed elevation $z_{b}$ as the only control variable, then for the joint identification of $z_{b}$ and initial conditions $\left(h^{0}, \mathbf{u}^{0}\right)$.

\subsection{Flow configuration}

We consider a $100 \times 16 m$ rectangular channel. The bed has a longitudinal slope of $0.4 \%$ and features a bump spanned on the whole width, centered in $x=40 \mathrm{~m}$ with an amplitude of $0.25 \mathrm{~m}$ and a length of $30 \mathrm{~m}$. This bump generates an acceleration of the flow in the region $x \in$ $[25,60]$.

A constant discharge $\bar{q}=8 \mathrm{~m}^{3} / \mathrm{s}$ is prescribed at the boundary $\Gamma_{q}: x=0 \mathrm{~m}$. The boundaries $y=0$ and $y=16 \mathrm{~m}$ are defined as walls and denoted by $\Gamma_{w}$. Finally, Neumann conditions are prescribed on the boundary $\Gamma_{t}: x=100 \mathrm{~m}$ (see Fig. 4 (a)). In order to simulate boundary layer effects, the value of the Manning coefficient $n$ is variable in space. In the central part of the domain, denoted by $\Omega_{1}$ and defined by $|y-8|<4 m$, $n$ is set to 0.02 . In the complement of $\Omega_{1}$ in $\Omega$, i.e. the lateral sides of the domain, denoted by $\Omega_{2}$, the value of

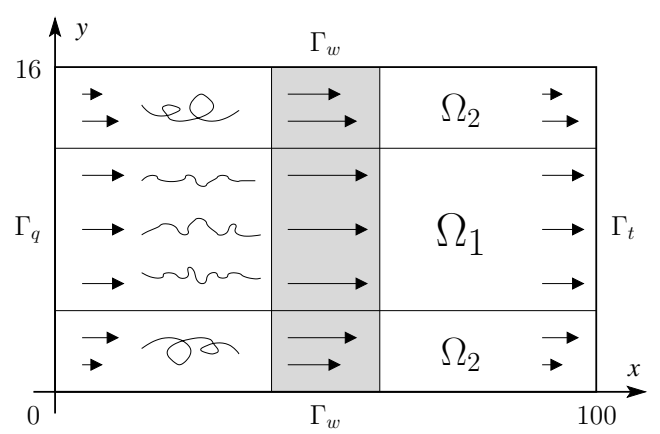

(a) Domain description

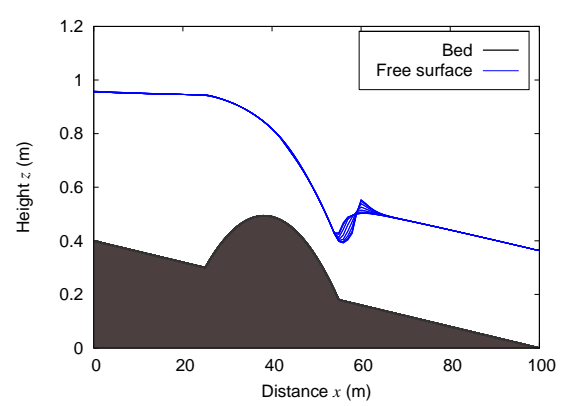

(b) Vertical cut of the fluid domain

Fig. 4 Twin experiments flow configuration 
$n$ increases linearly from 0.02 at the intersection with $\Omega_{1}$ up to 0.04 at the boundary $\Gamma_{w}$.

These conditions drive the flow to a steady state that is used as an initial condition for the twin experiments. A vertical cut of the fluid domain in the longitudinal plane in Fig. 4 (b) shows the bed and the free surface elevation for this configuration. The multiple lines that can be seen on the free surface correspond to different planes of the transversal discretization. Twin data assimilation experiments are carried out for a simulation time $T=$ $100 s$ and a constant time step $\Delta t=0.1 s$.

\subsection{Creation of observations}

Observations are created by the model from the reference steady flow described above. Water depth is recorded continuously in time at the abscissae $x_{1}=15 \mathrm{~m}$ and $x_{2}=$ $70 \mathrm{~m}$, for the whole width of the domain. These measurements are used as observations denoted by $h_{i}^{o b s}(y ; t)$ for $i=1,2$ in the twin experiments.

With regards to the creation of trajectories observations, virtual particles are dropped in the reference steady flow near boundary $\Gamma_{q}$ and transported by a turbulent surface velocity $\mathbf{v}^{\text {tr }}=\gamma \mathbf{u}+\widetilde{\mathbf{v}}$, where $\gamma=1$ and $\widetilde{\mathbf{v}}$ is a Gauss-Markov process as described in Section 6.2. The correlation matrix $R$ can be written as

$$
R=\left(\begin{array}{cc}
\sigma_{x}^{2} & \rho \sigma_{x} \sigma_{y} \\
\rho \sigma_{x} \sigma_{y} & \sigma_{y}^{2}
\end{array}\right)
$$

with standard deviations $\sigma_{x}$ and $\sigma_{y}$ defined as

$$
\sigma_{x}=\sqrt{\frac{c_{x}}{1+\left(u_{x}\right)^{2}}} \quad \text { and } \quad \sigma_{y}=\sqrt{\frac{c_{y}}{1+\left(u_{y}\right)^{2}}}
$$

Table 1 Values of Gauss-Markov process parameters.

\begin{tabular}{|c|c|c|c|c|}
\cline { 2 - 5 } \multicolumn{1}{c|}{} & $\rho$ & $c_{x}$ & $c_{y}$ & $T_{L}$ \\
\hline domain $\Omega_{1}$ & 0.2 & 0.5 & 0.3 & 0.6 \\
\hline domain $\Omega_{2}$ & 0.3 & 0.6 & 0.4 & 0.3 \\
\hline
\end{tabular}

This choice corresponds to a perturbation that lessens when the magnitude of the velocity increases. The numerical value of the parameters depends on the position in the domain. They are summarized in Table 1. A set of 32 particles is released in the flow, every 2 seconds of simulation, uniformly distributed over the width of the channel at the point $x=10 \mathrm{~m}$. Altogether, we take into account $N_{\text {obs }}=640$ particles. The trajectories of these particles transported by the turbulent velocity field $\mathbf{v}^{\text {tr }}$ are used as raw observations, denoted by $X_{i}^{\text {obs }}$. They are drawn with dotted lines in Fig. 5. Reference trajectories of particles that would be transported by an undisturbed velocity field (where $\widetilde{\mathbf{v}} \equiv 0$ ) are drawn in bold continuous lines. Such reference trajectories will be denoted by $X_{i}^{r e f}$ in the following. In the same way, the longitudinal and transversal velocities are traced respectively in Fig. 6 (a) and $6(\mathrm{~b})$.

\subsection{Trajectories filtering}

Filtered trajectories are reconstructed from the observed trajectories $X_{i}^{\text {obs }}$ using a time-space filter as described in Section 5. From the a priori hypothesis that the flow is stationary, we choose the largest possible time window $\mathcal{W}_{t}=[0, T]$ for all time $t$.

The dimensions of the space window should be chosen in order to be large enough to remove small-scale perturbations, yet small enough to prevent from smoothing most important characteristics of the flow. Since the flow features large longitudinal variations, the corresponding dimension of the space window is set to $1 \%$ of the domain length, i.e. $1 \mathrm{~m}$. Concerning the transversal dimension, a filter width equal to the size of the domain would remove boundary layer effects. On the other hand, a too small value would not smooth enough transversal perturbations. A good compromise has been found with $\frac{1}{16}$ of the channel width, i.e. $1 \mathrm{~m}$. A total of 200 filtered trajectories are reconstructed using sets of 10 particles released in the flow every 2 seconds, uniformly distributed over the width of the channel at the abscissa $x=10 \mathrm{~m}$.

\subsection{Identification of topography}

We seek to identify the reference topography used to create the observations, from the a priori hypothesis that the bed is made of a longitudinal slope of $0.4 \%$ without bump. To that purpose, we carry out data assimilation using the available observations, i.e. water depth measurements and particle trajectories. In this experiment, the only modified parameter when compared to the reference flow is the bed topography $z_{b}$. In particular, the initial condition remains unchanged.

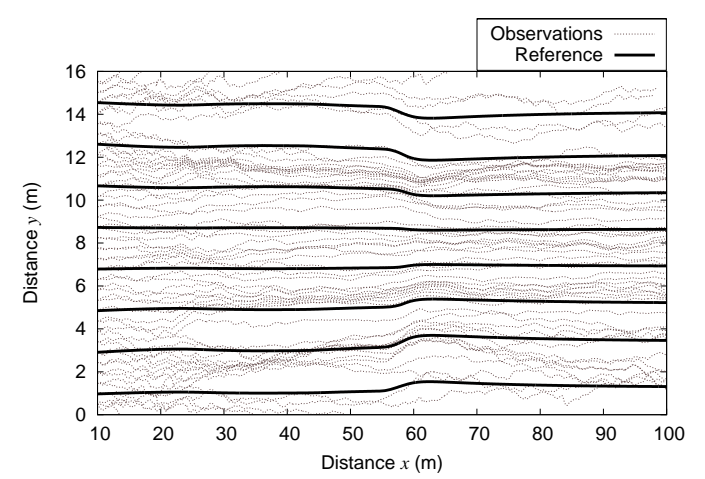

Fig. 5 Trajectories of particles transported by a turbulent surface velocity field. 


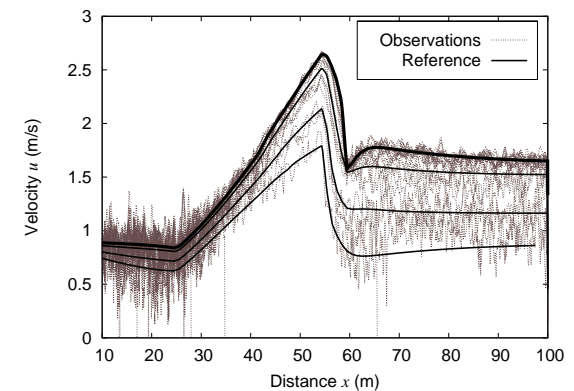

(a) Longitudinal velocity

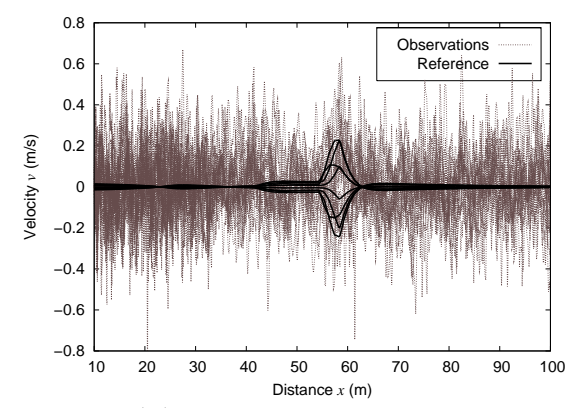

(b) Transversal velocity

Fig. 6 Velocities of particles transported by a turbulent surface velocity field.

\subsubsection{Water depth measurements}

We first try to identify the topography using only water depth measurements $h_{i}^{o b s}$ at the abscissae $x_{1}=15 \mathrm{~m}$ and $x_{2}=70 \mathrm{~m}$. The corresponding cost function is the following

$$
\begin{aligned}
j_{1}\left(z_{b}\right)=\frac{1}{2} \sum_{i=1}^{2} & \int_{0}^{T} \int_{0}^{y_{\max }}\left|h\left(x_{i}, y\right)-h_{i}^{o b s}(y)\right|^{2} d y d t \\
& +\frac{\alpha_{p}}{2}\left\|\nabla z_{b}\right\|_{\Omega}^{2} .
\end{aligned}
$$

A regularization term involving the norm of the topography gradient is introduced in the cost function in order to smooth the solution. The parameter $\alpha_{p}$ is chosen so that the weight of the penalization does not hinder the observations too much. In this experiment, it set to the value $\alpha_{p}=10^{-4}$.

In this configuration, the minimization algorithm converges very slowly to an identified topography that is not very satisfactory when compared to the reference. Figure 7 (a) presents the reference topography in a bold solid line as well as the identified one with a fine grey line. We can see that the latter comprises large variations with respect to the reference before the bump. In the figure to the right are shown the evolution of the cost function, the norm of its gradient and the value of $j_{\text {diag }}$. The decrease in the value of the diagnostic function $j_{\text {diag }}$ during the assimilation can be used as a measure of the quality of the identification. Here, we can see that it has not even be divided by 2 , which means that the identification is globally far from being satisfactory.

\subsubsection{Observation of reference trajectories}

In this experiment, we add observations of the 640 reference trajectories $X_{i}^{r e f}$ to the water depth measurements. The particles are transported by the undisturbed shallow water velocity field, so this experiment will be used as a reference to evaluate the performance of the filtering process in the following sections.

The cost function is built from $j_{1}$ with an additional term measuring the distance between virtual particles and reference particles.

$$
j_{2}\left(z_{b}\right)=j_{1}\left(z_{b}\right)+\frac{\alpha_{t}}{2} \sum_{i=1}^{N_{o b s}} \int_{0}^{T}\left|X_{i}(t)-X_{i}^{r e f}(t)\right|^{2} d t
$$

The parameter $\alpha_{t}$ is the weight given to the observations of trajectories. It is set to $\alpha_{t}=10^{-5}$, which roughly balances the value of the term associated to the water depth measurement and the one associated the observations of trajectories. The weight $\alpha_{p}$ remains set to $10^{-4}$. As we can see in Fig. 7 (b), the identified topography is very close to the reference. This observation is confirmed by the fact that the value of the diagnostic function $j_{\text {diag }}$ is divided by 530 at the end of the optimization process. The quality of the identification is thus very good.

\subsubsection{Observation of perturbed trajectories}

In this experiment, we combine observations of the 640 perturbed trajectories $X_{i}^{o b s}$ and water depth measurements. Therefore, the cost function is built from $j_{1}$ with an additional term measuring the distance between virtual particles and particles transported by the turbulent velocity field.

$$
j_{3}\left(z_{b}\right)=j_{1}\left(z_{b}\right)+\frac{\alpha_{t}}{2} \sum_{i=1}^{N_{o b s}} \int_{0}^{T}\left|X_{i}(t)-X_{i}^{o b s}(t)\right|^{2} d t
$$

When using the same values for the parameters $\alpha_{p}$ and $\alpha_{t}$ as in Section 7.4.2, the identified topography is very irregular with small-scale variations of large amplitude, as shown in Fig. 7 (c). Nevertheless, the shape of the bump is roughly identified. It can be noticed that the value of the diagnostic function $j_{\text {diag }}$ decreases in a first time during the minimization process, but then increases up to a quarter of its initial value.

It is possible to obtain slightly better results by increasing substantially the weight $\alpha_{p}$ of the regularization term. For instance, with $\alpha_{p}=10^{-2}$, the final value of $j_{\text {diag }}$ is divided by 7 with respect to its initial value instead of 4 . However, the identified topography is too much smoothed when compared to the reference. 

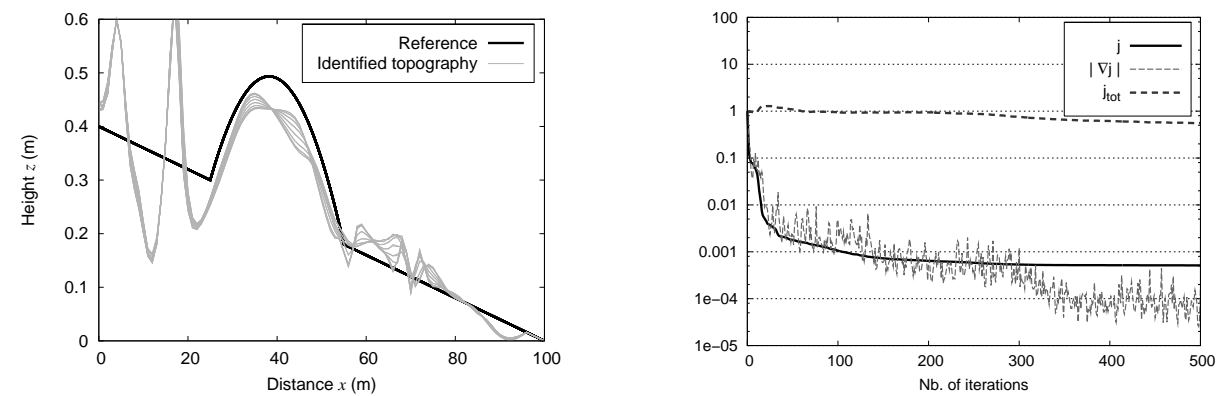

(a) Water depth measurements only. $\alpha_{p}=10^{-4}$.
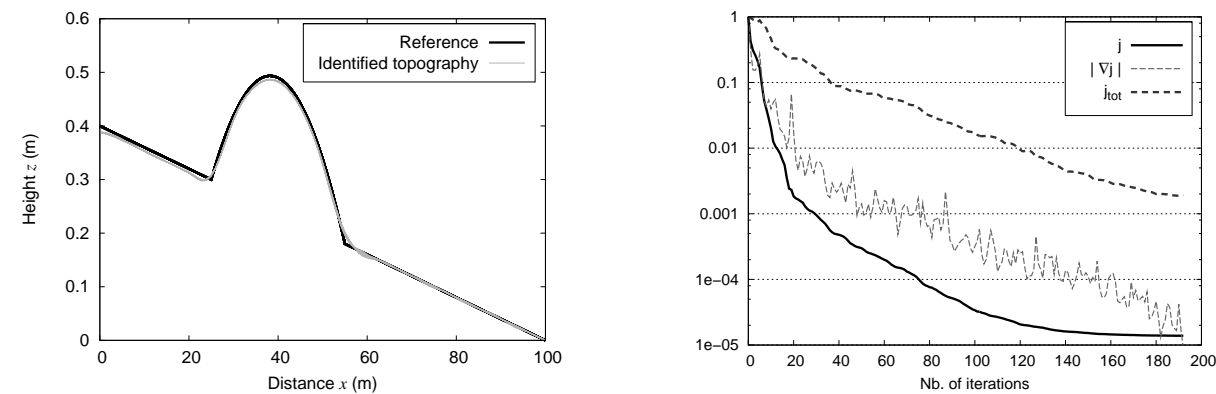

(b) Water depth measurements and reference trajectories. $\alpha_{t}=10^{-5}, \alpha_{p}=10^{-4}$.
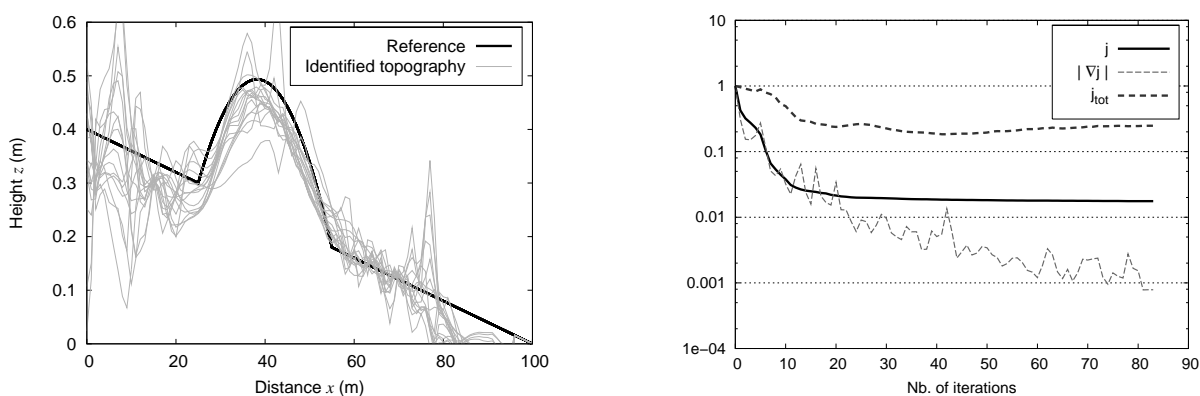

(c) Water depth measurements and perturbed trajectories. $\alpha_{t}=10^{-5}, \alpha_{p}=10^{-4}$.
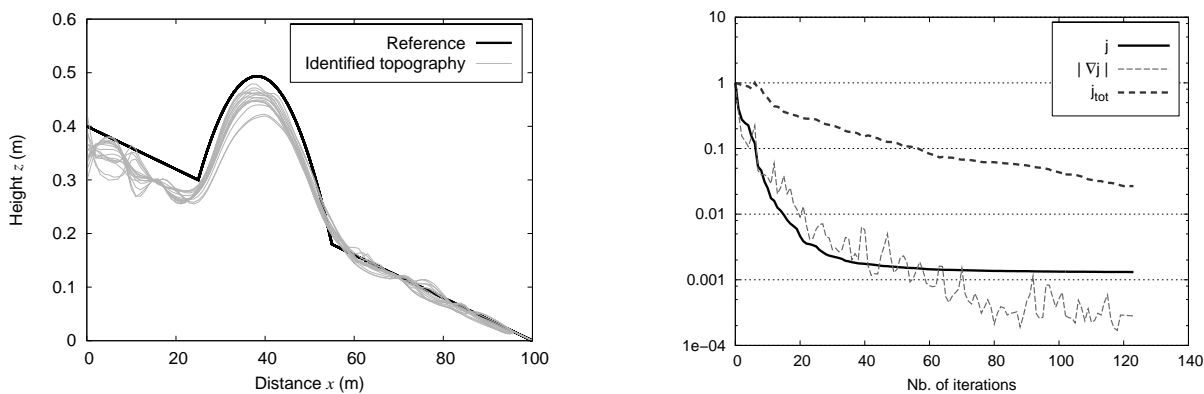

(d) Water depth measurements and filtered trajectories. $\alpha_{t}=2 \times 10^{-5}, \alpha_{p}=10^{-3}$.

Fig. 7 Identification of the topography using water depth measurements and trajectories. Left column: identified topography. Right column: convergence of the cost function.

\subsubsection{Observation of filtered trajectories}

We now use the filtered trajectories $\bar{X}_{j}$ as observations. The cost function is built from $j_{1}$, with an additional term measuring the distance between the trajectories of virtual particles and $N_{m}$ filtered trajectories.

$$
j_{4}\left(z_{b}\right)=j_{1}\left(z_{b}\right)+\frac{\alpha_{t}}{2} \sum_{j=1}^{N_{m}} \int_{0}^{T}\left|X_{j}(t)-\bar{X}_{j}(t)\right|^{2} d t
$$

As described in Section 7.2, we have $N_{m}=200$. For the weight parameters, we choose $\alpha_{t}=2 \times 10^{-5}$ and 
$\alpha_{p}=10^{-3}$. Figure $7(\mathrm{~d})$ shows a substantial improvement in the quality of topography identification. Unlike the case with unfiltered trajectories, the value of the diagnostic function $j_{\text {diag }}$ regularly decreases all along the minimization process. Finally, it is divided by 37 with respect to its initial value.

\subsection{Joint identification of topography and initial conditions}

We now seek to identify jointly the topography $z_{b}$ and the initial conditions (water depth $h^{0}$ and velocity $\mathbf{u}^{0}$ ) using all available observations from the initial assumption on both parameters. As in Section 7.4, we make the a priori hypothesis that the bed is made of a longitudinal slope of $0.4 \%$ without bump. However, regarding the initial condition, we use the steady state obtained with the modified topography instead of the reference one.

The filtered trajectories $\bar{X}_{j}$ are used as observations in addition to the water depth measurements. The cost function is similar to $j_{4}$ in Section 7.4.4. The only difference is that the initial condition $h^{0}$ and $\mathbf{u}^{0}$ are now control variables. The new cost function can then be written as

$$
\begin{aligned}
& j_{5}\left(z_{b}, h^{0}, \mathbf{u}^{0}\right)= \\
& \frac{1}{2} \sum_{i=1}^{2} \int_{0}^{T} \int_{0}^{y_{\max }}\left|h\left(x_{i}, y\right)-h_{i}^{o b s}(y)\right|^{2} d y d t \\
& \quad+\frac{\alpha_{t}}{2} \sum_{j=1}^{N_{m}} \int_{0}^{T}\left|X_{j}(t)-\bar{X}_{j}(t)\right|^{2} d t \\
& +\frac{\alpha_{p}}{2}\left\|\nabla z_{b}\right\|_{\Omega}^{2}
\end{aligned}
$$

The weight given to the observations of trajectories is set to $\alpha_{t}=10^{-4}$ while the one for the regularization term is set to $\alpha_{p}=8 \times 10^{-3}$. As shown in Fig. 8 (a), the identified topography is close to the reference, with a good recovery of the bump. It is similar to the result in Section 7.4.4. As for the initial conditions, we can see in Fig. 8 (c) and (d) that it reproduces the same main features as the reference. However, we can notice irregular variations in the identified variables in the upper part of the flow, as well as a slight over-estimation of the water depth combined with a slight underestimation of the longitudinal velocity in the area just downstream from the bump.

The value of the diagnostic function $j_{\text {diag }}$ is divided by about 20 at the end of the optimization process with respect to its initial value.

\section{Conclusion}

We have presented a method to include lagrangian observations into a variational data assimilation framework in order to improve the identification of control variables for a river hydraulics model based on the shallow water equations. The link between the eulerian state variables from the shallow water model and the lagrangian observations of particle trajectories is made thanks to a weakly coupled transport model. Numerical twin data assimilation experiments demonstrate that this method makes it possible to improve the identification of bed elevation and initial conditions significantly in an academic configuration.

A simple spatio-temporal filter has been used successfully to improve the quality of the identification with trajectories observations transported by a velocity field with small-scale perturbations.

In order to improve identification, a regularization term on the gradient of topography was introduced in the cost function. The initial condition may be similarly penalized in order to prevent large oscillations from arising in the solution, as observed in the last experiment concerning the joint identification of bed elevation and initial conditions. Another option would rely on an appropriate parameterization of the control variables in order to reduce the dimensions of the control space.

The application of the method to real data collected from video images of a steady flow in a laboratory channel is being conducted.

\section{References}

1. Atanov, G.A., Evseeva, E.G., Meselhe, E.A.: Estimation of roughness profile in trapezoidal open channels. Journal of Hydraulic Engineering 125(3), 309-312 (1999)

2. Bélanger, E., Vincent, A.: Data assimilation (4D-VAR) to forecast flood in shallow-waters with sediment erosion. Journal of Hydrology 300(1-4), 114-125 (2005)

3. Carter, E.F.: Assimilation of lagrangian data into a numerical model. Dynamics of Atmospheres and Oceans 13(3-4), 335-348 (1989)

4. Courtier, P., Talagrand, O.: Variational assimilation of meteorological observations with the direct and adjoint shallow water equations. Tellus 42(A), 531-549 (1990)

5. Creutin, J.D., Muste, M., Bradley, A.A., Kim, S.C., Kruger, A.: River gauging using PIV techniques: a proof of concept experiment on the Iowa River. Journal of Hydrology 277, 182-194 (2003)

6. Ding, Y., Jia, Y., Wang, S.S.Y.: Identification of Manning's roughness coefficients in shallow water flows. Journal of Hydraulic Engineering 130(6), 501-510 (2004)

7. Gilbert, J.C., Lemaréchal, C.: Some numerical experiments with variable storage quasi-Newton algorithms. Mathematical programming 45, 407-435 (1989)

8. Griewank, A.: Evaluating Derivatives: Principles and Techniques of Algorithmic Differentiation, Frontiers in Appl. Math., vol. 19. SIAM (2000)

9. Hartnack, J., Madsen, H.: Data assimilation in river flow modelling. In: $4^{\text {th }}$ DHI software conference, 6-8 june 2001. Scanticon Conference Center, Helsingør, Danemark (2001)

10. Hascoët, L., Pascual, V.: TAPEnade 2.1 user's guide. Technical Report RT-300, INRIA (2004)

11. Honnorat, M., Le Dimet, F.X., Loukili, Y., Monnier, J.: DASSFLOW : a direct and adjoint model for $2 \mathrm{~d}$ shallow water flows. Research Report RR-5756, INRIA (2005) 


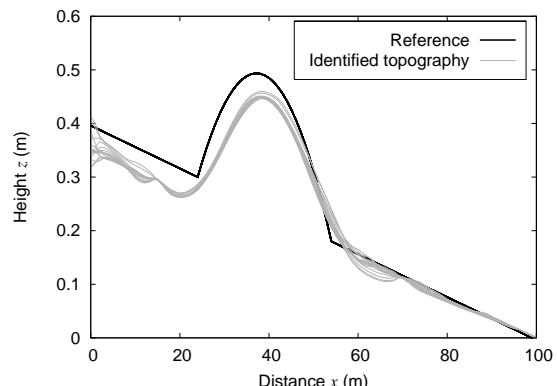

(a) Identified topography

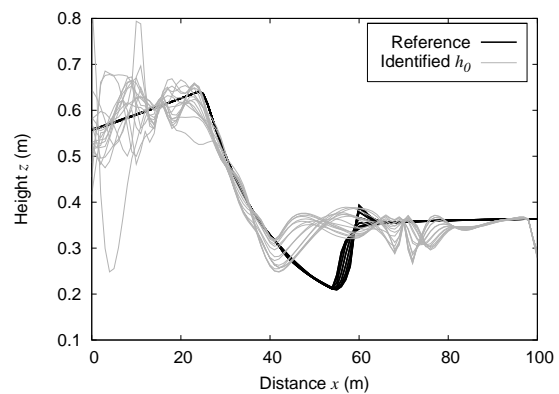

(c) Identified $h^{0}$

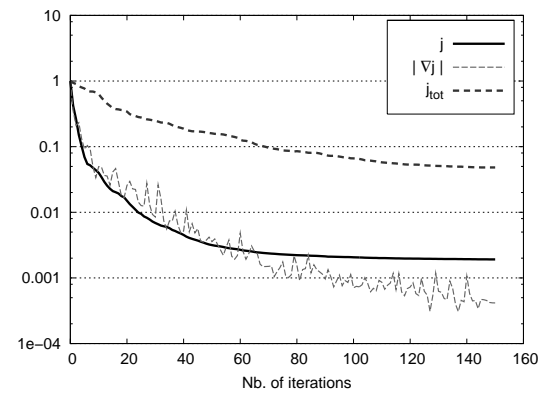

(b) Evolution of the cost function

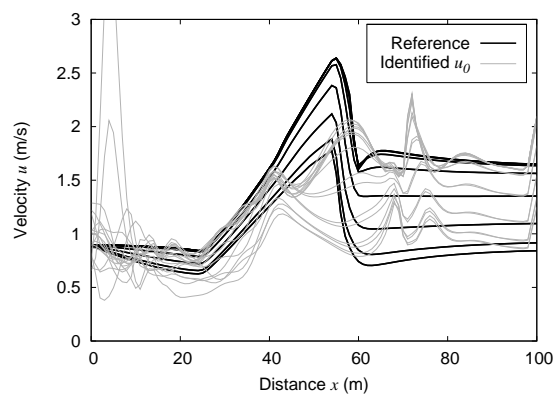

(d) Identified $u^{0}$

Fig. 8 Joint identification of the topography and the initial conditions using water depth measurements and filtered trajectories. $\alpha_{t}=1 \times 10^{-4}, \alpha_{p}=8 \times 10^{-3}$.

12. Kamachi, M., O'Brien, J.J.: Continuous data assimilation of drifting buoy trajectory into an equatorial Pacific Ocean model. Journal of Marine Systems 6, 159-178 (1995)

13. Khatibi, R.H., Williams, J.J.R., Wormleaton, P.R.: Identification problem of open-channel friction parameters. Journal of Hydraulic Engineering 123(12), 1078-1088 (1997)

14. Kuznetsov, L., Ide, K., Jones, C.K.R.T.: A method for assimilation of lagrangian data. Monthly Weather Review 131(10), 2247-2260 (2003)

15. Le Dimet, F.X., Talagrand, O.: Variational algorithms for analysis and assimilation of meteorological observations: theoretical aspects. Tellus 38(A), 97-110 (1986)

16. LeVeque, R.J.: Balancing source terms and flux gradients in high-resolution Godunov methods: The quasi-steady wave-propagation algorithm. Journal of Computational Physics 146, 346-365 (1998)

17. Lions, J.L.: Optimal control of systems governed by partial differential equations. Springer-Verlag (1971)

18. Madsen, H., Hartnack, J., Sørensen, J.V.T.: Data assimilation in a flood modelling system using the Ensemble Kalman filter. In: CMWR-Xvi. Copenhaguen, Danemark (2006)

19. Mazauric, C.: Assimilation de données pour les modèles d'hydraulique fluviale. Estimation de paramètres, analyse de sensibilité et décomposition de domaine. Ph.D. thesis, Université Joseph Fourier, LMC-IMAG (2003). In french

20. Muste, M., Xiong, Z., Schöne, J., Li, Z.: Validation and extension of image velocimetry capabilities for flow diagnostic in hydraulic modeling. Journal of Hydraulic Engineering 130(3), 175-185 (2004)

21. Nodet, M.: Variational assimilation of lagrangian data in oceanography. Inverse Problems 22, 245-263 (2006)

22. Roux, H., Dartus, D.: Data assimilation applied to hydraulic parameter identification. In: British hydrological society international conference 2004, hydrology: science and practice for the 21st century, pp. 354-361. London, United Kingdom (2004)

23. Salman, H., Kuznetsov, L., Jones, C.K.R.T.: A method for assimilating lagrangian data into a shallow-waterequation ocean model. Monthly Weather Review 134(4), 1081-1101 (2006)

24. Talagrand, O. Courtier, P. Variational assimilation of meteorological observations with the adjoint vorticity equation. I : Theory. Quarterly Journal of the Royal Meteorological Society 113(1311-1328) (1987)

25. Toro, E.F.: Shock-capturing methods for free-surface shallow flows. J. Wiley and Sons (2001)

26. Yang, J.: Assimilation de données variationnelle pour les problèmes de transport des sédiments en rivière. $\mathrm{Ph}$.D. thesis, Université Joseph Fourier, LMC-IMAG (1999). In french 